CRYSTALLOGRAPHIC COMMUNICATIONS

ISSN 2056-9890

Received 4 February 2015

Accepted 18 February 2015

Edited by A. J. Lough, University of Toronto, Canada

Keywords: crystal structure; $\pi-\pi$ interactions; organoselenium compounds

CCDC reference: 1050354

Supporting information: this article has supporting information at journals.iucr.org/e

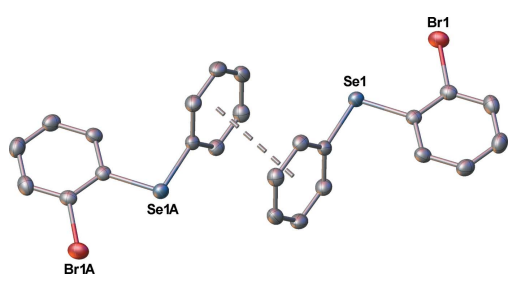

OPEN $\odot$ ACCESS

\section{Crystal structure of 1-bromo-2-(phenylselenyl)- benzene}

\author{
Bronte J. Charette ${ }^{a, b}$ and Jamie S. Ritch ${ }^{a, b *}$
}

aDepartment of Chemistry, The University of Winnipeg, 515 Portage Avenue, Winnipeg, MB, R3B 2E9, Canada, and bepartment of Chemistry, 360 Parker Building, University of Manitoba, Winnipeg, MB, R3T 2N2, Canada.

*Correspondence e-mail: j.ritch@uwinnipeg.ca

In the title compound, $\mathrm{C}_{12} \mathrm{H}_{9} \mathrm{BrSe}$, the Se atom exhibits a bent geometry, with a $\mathrm{C}-\mathrm{Se}-\mathrm{C}$ bond angle of $99.19(6)^{\circ}$. The ortho $\mathrm{Se}$ and $\mathrm{Br}$ atoms are slightly displaced from opposite faces of the mean plane of the benzene ring [by 0.129 (2) and 0.052 (2) $\AA$, respectively]. The planes of the benzene and phenyl rings form a dihedral angle of $72.69(5)^{\circ}$. In the crystal, $\pi$-stacking interactions between inversion-related phenyl rings are observed, with a centroid-centroid distance of 3.630 (1) $\AA$.

\section{Chemical context}

Organoselenium compounds have been found to have diverse scientific applications. For instance, the antioxidant capabilities of the glutathione peroxidases has inspired the synthesis of selenium-containing enzyme mimetics for therapeutic use (Schewe, 1995), and examples are known of selenium-based conjugated materials exhibiting superconductivity (Jérome $e t$ al., 1980). Our research group is interested in organoselenium compounds in the context of designing ligands for coordination to transition metals to generate catalytic complexes. This is an area of growing interest, as examples of seleniumcontaining catalysts with higher activity than the ubiquitous phosphine analogues are discovered (Kumar et al., 2012). The title compound represents a potentially valuable starting material for the synthesis of ligands containing $-\mathrm{SePh}$ donor groups, as the ortho- $\mathrm{Br}$ atom provides a site of functionalization via, for example, lithium halogen exchange followed by electrophile addition, or a metal-catalyzed cross-coupling reaction. Though previously prepared (Cristau et al., 1985), its structure has remained unreported.

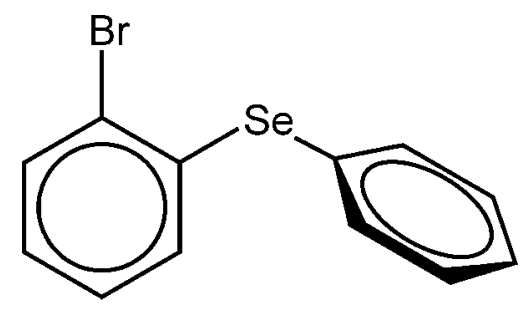

\section{Structural commentary}

The molecular structure of the title compound, (I), is depicted in Fig. 1. The asymmetric unit possesses one complete molecule, which features no disorder. The central Se atom exhibits a bent geometry $\left[\mathrm{C} 1-\mathrm{Se} 1-\mathrm{C} 7=99.19(6)^{\circ}\right]$. The two planes comprising the benzene and phenyl ring $\mathrm{C}$ atoms are twisted 


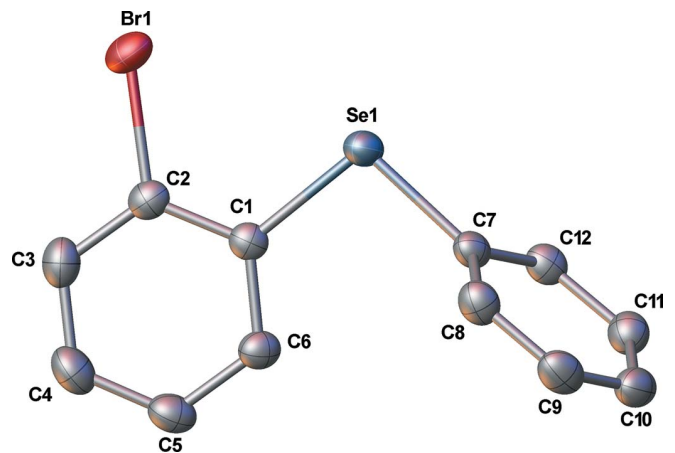

Figure 1

The molecular structure of the title compound, (I), showing $50 \%$ probability ellipsoids.

by $72.69(5)^{\circ}$ relative to each other. The $\mathrm{Br}$ and Se atoms are twisted with respect to the disubstituted benzene ring, as evidenced by displacements in opposite directions from the mean plane of the ring by 0.052 (2) and 0.129 (2) $\AA$, respectively, and the torsion angle $\mathrm{Br} 1-\mathrm{C} 2-\mathrm{C} 1-\mathrm{Se} 1$ is $4.2(1)^{\circ}$.

The $\mathrm{Se}-\mathrm{C}$ distances of 1.9171 (14) and 1.9198 (14) $\AA$ are equal within experimental error. At 1.9044 (14) $\AA$, the $\mathrm{C}-\mathrm{Br}$ distance is measurably shorter than the $\mathrm{Se}-\mathrm{C}$ bond lengths.

\section{Supramolecular features}

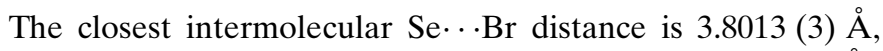
which lies outside the sum of the van der Waals radii (3.75 $\AA$ ) for these two elements (Bondi, 1964). The phenyl group of each molecule is associated with the same group on an adjacent molecule by a slipped $\pi$-stacking interaction (Fig. 2). The two molecules in the dimeric units are situated about a crystallographic inversion centre. The centroid-to-centroid separation of the aromatic rings is 3.630 (1) $\AA$, while the nearest centroid-to-plane distance is 3.378 (1) $\AA$. Together, these are indicative of the slipped nature of the $\pi-\pi$ interaction. The ring separation is in the normal range ( $c a$ 3.3$3.8 \AA$ ) for $\pi$-stacked interactions (Janiak, 2000). The packing is illustrated in Fig. 3.

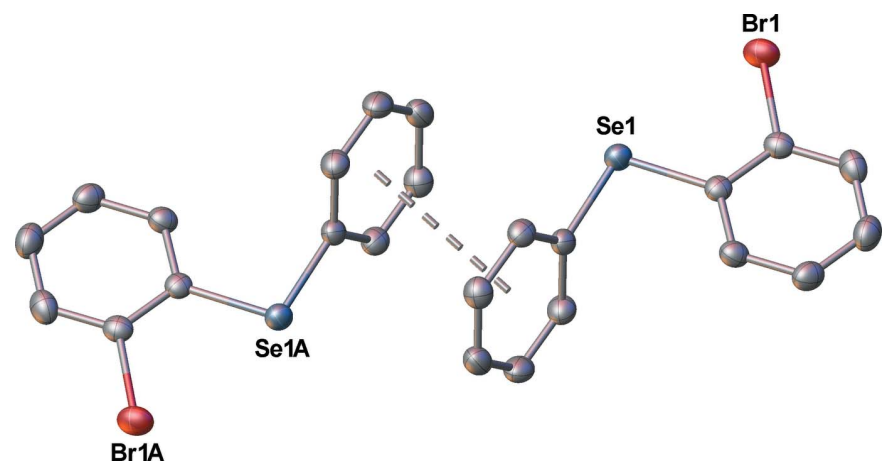

Figure 2

Slipped $\pi$-stacked dimers of 1-bromo-2-(phenylselenyl)benzene. Each molecule is related to the other by an inversion centre at the centre of the centroid-centroid line.

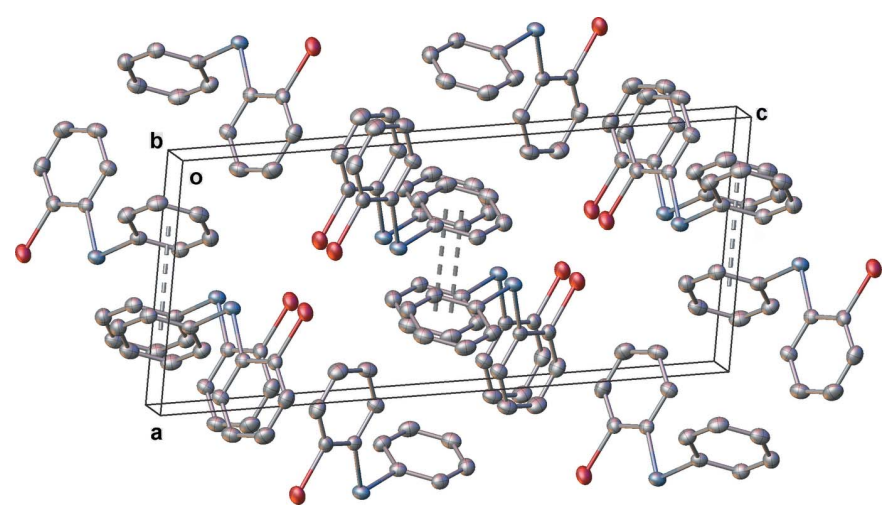

Figure 3

Packing diagram for (I), viewed along the crystallographic $b$ axis.

\section{Database survey}

A search of the Cambridge Structural Database (CSD, Version 5.35; Groom \& Allen, 2014) reveals 172 structures featuring two-coordinate aryl-substituted selenium centres. The mean bond angle of $98(4)^{\circ}$ and $\mathrm{Se}-\mathrm{C}$ (aryl) distance of 1.92 (2) $\AA$ for these structures match well with the parameters observed for 1-bromo-2-(phenylselenyl)benzene.

Only two structures in the CSD feature the title compound as a substructure: bis(2-bromo-4,5-dimethoxyphenyl) selenide (SAKBIP; Schiffling and Klar, 1989) and 1,4-dibromo-2,3,5,6tetrakis(phenylseleno)benzene (MUHTOZ; Sato \& Kanatomi, 2009). Both of these compounds exhibit similar twisted orientations of the two aromatic rings, but lack $\pi$-stacking secondary bonding interactions, presumably due to their highly substituted nature. By contrast, the structure of a less sterically crowded analogue, 1-bromo-8-(phenylselenyl)naphthalene (CIKPUI; Fuller et al., 2007), exhibits slipped $\pi$-stacking of the naphthalene rings.

\section{Synthesis and crystallization}

1-Bromo-2-(phenylselenyl)benzene has been prepared in previous reports using several methodologies, including nickel(II)-catalyzed coupling of $\mathrm{NaSePh}$ with 1,2-dibromobenzene (Cristau et al., 1985) and the copper-catalyzed coupling of diphenyl diselenide with 1-bromo-2-iodobenzene (Dandapat et al., 2011), which is the procedure followed for this study (Fig. 4). Purification via flash column chromatography with a silica stationary phase was conducted as reported. Though described by Dandapat et al. (2011) as being a 'slightly brown oil', we found that this compound was a nearly

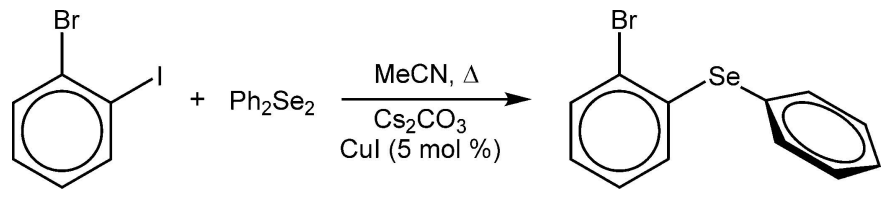

Figure 4

The synthetic route to 1-bromo-2-(phenylselenyl)benzene, (I). 
Table 1

Experimental details.

\begin{tabular}{|c|c|}
\hline \multicolumn{2}{|l|}{ Crystal data } \\
\hline Chemical formula & $\mathrm{C}_{12} \mathrm{H}_{9} \mathrm{BrSe}$ \\
\hline$M_{\mathrm{r}}$ & 312.06 \\
\hline Crystal system, space group & Monoclinic, $P 2_{1} / c$ \\
\hline Temperature (K) & 173 \\
\hline$a, b, c(\AA)$ & $8.1171(4), 7.6028(4), 18.1345(10)$ \\
\hline$\beta\left(^{\circ}\right)$ & $99.2668(6)$ \\
\hline$V\left(\AA^{3}\right)$ & $1104.52(10)$ \\
\hline$Z$ & 4 \\
\hline Radiation type & Мо $K \alpha$ \\
\hline$\mu\left(\mathrm{mm}^{-1}\right)$ & 6.97 \\
\hline Crystal size (mm) & $0.35 \times 0.32 \times 0.26$ \\
\hline \multicolumn{2}{|l|}{ Data collection } \\
\hline Diffractometer & Bruker APEXII CCD \\
\hline Absorption correction & $\begin{array}{l}\text { Numerical ( } S A D A B S \text {; Bruker, } \\
\text { 2013) }\end{array}$ \\
\hline$T_{\min }, T_{\max }$ & $0.205,0.361$ \\
\hline $\begin{array}{l}\text { No. of measured, independent and } \\
\text { observed }[I>2 \sigma(I)] \text { reflections }\end{array}$ & $21749,2742,2528$ \\
\hline$R_{\text {int }}$ & 0.016 \\
\hline$(\sin \theta / \lambda)_{\max }\left(\AA^{-1}\right)$ & 0.669 \\
\hline \multicolumn{2}{|l|}{ Refinement } \\
\hline$R\left[F^{2}>2 \sigma\left(F^{2}\right)\right], w R\left(F^{2}\right), S$ & $0.017,0.042,1.06$ \\
\hline No. of reflections & 2742 \\
\hline No. of parameters & 127 \\
\hline $\mathrm{H}$-atom treatment & $\mathrm{H}$-atom parameters constrained \\
\hline$\Delta \rho_{\max }, \Delta \rho_{\min }\left(\mathrm{e} \AA^{-3}\right)$ & $0.28,-0.45$ \\
\hline
\end{tabular}

Computer programs: APEX2 and SAINT (Bruker, 2013), SHELXS97 (Sheldrick, 2008), SHELXL2014 (Sheldrick, 2015) and OLEX2 (Dolomanov et al., 2009).

colourless liquid which slowly crystallized upon standing at room temperature. NMR spectroscopic analysis matched the reported data.

Though quite soluble in common solvents, including nonpolar solvents such as hexanes, in the highly lipophilic hexamethyldisiloxane we found this substance was only moderately soluble. It crystallized readily as transparent colourless crystals from a solution in this solvent upon storage at $273 \mathrm{~K}$.

\section{Refinement}

Crystal data, data collection and structure refinement details are summarized in Table 1. No special considerations were needed for the refinement. $\mathrm{H}$ atoms were placed in calculated positions, with $\mathrm{C}-\mathrm{H}=0.95 \AA$ and $U_{\text {iso }}(\mathrm{H})=1.2 U_{\text {eq }}(\mathrm{C})$, and treated in a riding-model approximation.

\section{Acknowledgements}

Funding from The University of Winnipeg is gratefully acknowledged. The authors thank Bob McDonald (X-Ray Crystallography Laboratory, Department of Chemistry, University of Alberta, Edmonton, Canada) for the collection of X-ray diffraction data, and the University of Manitoba, Department of Chemistry, for an adjunct appointment (JSR).

\section{References}

Bondi, A. (1964). J. Phys. Chem. 68, 441-451.

Bruker (2013). APEX2, SAINT and SADABS. Bruker AXS Inc., Madison, Wisconsin, USA.

Cristau, H. J., Chabaud, B., Labaudiniere, R. \& Christol, H. (1985). Organometallics, 4, 657-661.

Dandapat, A., Korupalli, C., Prasad, D. J. C., Singh, R. \& Sekar, G. (2011). Synthesis, pp. 2297-2302.

Dolomanov, O. V., Bourhis, L. J., Gildea, R. J., Howard, J. A. K. \& Puschmann, H. (2009). J. Appl. Cryst. 42, 339-341.

Fuller, A. L., Knight, F. R., Slawin, A. M. Z. \& Woollins, J. D. (2007). Acta Cryst. E63, o3855.

Groom, C. R. \& Allen, F. H. (2014). Angew. Chem. Int. Ed. 53, 662 671.

Janiak, C. (2000). J. Chem. Soc. Dalton Trans. pp. 3885-3896.

Jérome, D., Mazaud, A., Ribault, M. \& Bechgaard, K. (1980). J. Phys. Lett. 41, 95-98.

Kumar, A., Rao, G. K., Saleem, F. \& Singh, A. K. (2012). Dalton Trans. 41, 11949-11977.

Sato, M. \& Kanatomi, Y. (2009). J. Sulfur Chem. 30, 469-476.

Schewe, T. (1995). Gen. Pharmacol. 26, 1153-1169.

Schiffling, C. \& Klar, G. (1989). J. Chem. Res. pp. 2-3.

Sheldrick, G. M. (2008). Acta Cryst. A64, 112-122.

Sheldrick, G. M. (2015). Acta Cryst. C71, 3-8. 


\section{supporting information}

Acta Cryst. (2015). E71, 327-329 [doi:10.1107/S205698901500345X]

\section{Crystal structure of 1-bromo-2-(phenylselenyl)benzene}

\section{Bronte J. Charette and Jamie S. Ritch}

\section{Computing details}

Data collection: APEX2 (Bruker 2013); cell refinement: SAINT (Bruker, 2013); data reduction: SAINT (Bruker, 2013); program(s) used to solve structure: SHELXS97 (Sheldrick, 2008); program(s) used to refine structure: SHELXL2014 (Sheldrick, 2015); molecular graphics: OLEX2 (Dolomanov et al., 2009); software used to prepare material for publication: OLEX2 (Dolomanov et al., 2009).

\section{1-Bromo-2-(phenylselenyl)benzene}

Crystal data

$\mathrm{C}_{12} \mathrm{H}_{9} \mathrm{BrSe}$

$M_{r}=312.06$

Monoclinic, $P 2_{1} / c$

$a=8.1171$ (4) $\AA$

$b=7.6028(4) \AA$

$c=18.1345(10) \AA$

$\beta=99.2668(6)^{\circ}$

$V=1104.52(10) \AA^{3}$

$Z=4$

Data collection

Bruker APEXII CCD

diffractometer

$\omega$ scans

Absorption correction: numerical

(SADABS; Bruker, 2013)

$T_{\min }=0.205, T_{\max }=0.361$

21749 measured reflections

\section{Refinement}

Refinement on $F^{2}$

Least-squares matrix: full

$R\left[F^{2}>2 \sigma\left(F^{2}\right)\right]=0.017$

$w R\left(F^{2}\right)=0.042$

$S=1.06$

2742 reflections

127 parameters

0 restraints
$F(000)=600$

$D_{\mathrm{x}}=1.877 \mathrm{Mg} \mathrm{m}^{-3}$

Mo $K \alpha$ radiation, $\lambda=0.71073 \AA$

Cell parameters from 9910 reflections

$\theta=2.3-28.2^{\circ}$

$\mu=6.97 \mathrm{~mm}^{-1}$

$T=173 \mathrm{~K}$

Fragment, colourless

$0.35 \times 0.32 \times 0.26 \mathrm{~mm}$

2742 independent reflections

2528 reflections with $I>2 \sigma(I)$

$R_{\text {int }}=0.016$

$\theta_{\text {max }}=28.4^{\circ}, \theta_{\min }=2.3^{\circ}$

$h=-10 \rightarrow 10$

$k=-10 \rightarrow 10$

$l=-24 \rightarrow 24$

Primary atom site location: structure-invariant direct methods

Hydrogen site location: inferred from neighbouring sites

$\mathrm{H}$-atom parameters constrained

$w=1 /\left[\sigma^{2}\left(F_{\mathrm{o}}^{2}\right)+(0.0194 P)^{2}+0.5087 P\right]$

where $P=\left(F_{\mathrm{o}}{ }^{2}+2 F_{\mathrm{c}}{ }^{2}\right) / 3$

$(\Delta / \sigma)_{\max }=0.001$

$\Delta \rho_{\max }=0.28 \mathrm{e} \AA^{-3}$

$\Delta \rho_{\min }=-0.45$ e $\AA^{-3}$ 
Special details

Experimental. The following wavelength and cell were deduced by $S A D A B S$ from the direction cosines etc. They are given here for emergency use only: CELL 0.71074 8.140 7.626 18.183 89.999 99.279 90.004.

Geometry. All e.s.d.'s (except the e.s.d. in the dihedral angle between two 1.s. planes) are estimated using the full covariance matrix. The cell e.s.d.'s are taken into account individually in the estimation of e.s.d.'s in distances, angles and torsion angles; correlations between e.s.d.'s in cell parameters are only used when they are defined by crystal symmetry. An approximate (isotropic) treatment of cell e.s.d.'s is used for estimating e.s.d.'s involving 1.s. planes.

Fractional atomic coordinates and isotropic or equivalent isotropic displacement parameters $\left(\AA^{2}\right)$

\begin{tabular}{lllll}
\hline & $x$ & $y$ & $z$ & $U_{\text {iso }} / U_{\text {eq }}$ \\
\hline Br1 & $0.37269(2)$ & $0.33279(2)$ & $0.26417(2)$ & $0.03601(5)$ \\
Se1 & $0.39491(2)$ & $0.65383(2)$ & $0.38572(2)$ & $0.02860(5)$ \\
C1 & $0.19202(17)$ & $0.52181(18)$ & $0.36221(7)$ & $0.0237(3)$ \\
C2 & $0.18210(18)$ & $0.39017(19)$ & $0.30840(8)$ & $0.0266(3)$ \\
C3 & $0.0361(2)$ & $0.2971(2)$ & $0.28518(9)$ & $0.0349(3)$ \\
H3 & 0.0323 & 0.2087 & 0.2479 & $0.042^{*}$ \\
C4 & $-0.1043(2)$ & $0.3346(2)$ & $0.31696(10)$ & $0.0384(4)$ \\
H4 & -0.2058 & 0.2735 & 0.3008 & $0.046^{*}$ \\
C5 & $-0.09621(19)$ & $0.4610(2)$ & $0.37222(9)$ & $0.0341(3)$ \\
H5 & -0.1918 & 0.4846 & 0.3947 & $0.041^{*}$ \\
C6 & $0.05039(18)$ & $0.55397(19)$ & $0.39518(8)$ & $0.0283(3)$ \\
H6 & 0.0546 & 0.6399 & 0.4335 & $0.034^{*}$ \\
C7 & $0.32416(18)$ & $0.82409(18)$ & $0.45228(8)$ & $0.0255(3)$ \\
C8 & $0.22331(19)$ & $0.9649(2)$ & $0.42396(9)$ & $0.0315(3)$ \\
H8 & 0.1849 & 0.9747 & 0.3718 & $0.038^{*}$ \\
C9 & $0.17967(19)$ & $1.0906(2)$ & $0.47282(10)$ & $0.0350(3)$ \\
H9 & 0.1097 & 1.1861 & 0.4541 & $0.042^{*}$ \\
C10 & $0.23779(19)$ & $1.0773(2)$ & $0.54880(10)$ & $0.0343(3)$ \\
H10 & 0.2074 & 1.1636 & 0.5820 & $0.041^{*}$ \\
C11 & $0.3399(2)$ & $0.9387(2)$ & $0.57634(9)$ & $0.0325(3)$ \\
H11 & 0.3809 & 0.9311 & 0.6283 & $0.039^{*}$ \\
C12 & $0.38282(18)$ & $0.81058(19)$ & $0.52816(9)$ & $0.0283(3)$ \\
H12 & 0.4518 & 0.7145 & 0.5471 & $0.034^{*}$ \\
& & & & \\
\hline
\end{tabular}

Atomic displacement parameters $\left(\AA^{2}\right)$

\begin{tabular}{lllllll}
\hline & $U^{11}$ & $U^{22}$ & $U^{33}$ & $U^{12}$ & $U^{13}$ & $U^{23}$ \\
\hline Br1 & $0.03941(9)$ & $0.04133(10)$ & $0.02890(8)$ & $0.00838(7)$ & $0.01038(6)$ & $-0.00290(6)$ \\
Se1 & $0.02379(8)$ & $0.02797(8)$ & $0.03495(9)$ & $-0.00057(5)$ & $0.00750(6)$ & $-0.00292(6)$ \\
C1 & $0.0245(6)$ & $0.0210(6)$ & $0.0253(6)$ & $0.0015(5)$ & $0.0034(5)$ & $0.0045(5)$ \\
C2 & $0.0294(7)$ & $0.0261(7)$ & $0.0244(6)$ & $0.0041(6)$ & $0.0045(5)$ & $0.0030(5)$ \\
C3 & $0.0418(9)$ & $0.0299(8)$ & $0.0310(8)$ & $-0.0032(7)$ & $-0.0002(6)$ & $-0.0033(6)$ \\
C4 & $0.0322(8)$ & $0.0352(9)$ & $0.0460(9)$ & $-0.0086(7)$ & $0.0010(7)$ & $0.0016(7)$ \\
C5 & $0.0274(7)$ & $0.0312(8)$ & $0.0449(9)$ & $-0.0010(6)$ & $0.0098(6)$ & $0.0047(7)$ \\
C6 & $0.0292(7)$ & $0.0230(7)$ & $0.0340(7)$ & $0.0014(6)$ & $0.0089(6)$ & $0.0009(6)$ \\
C7 & $0.0228(6)$ & $0.0208(6)$ & $0.0332(7)$ & $-0.0020(5)$ & $0.0049(5)$ & $-0.0010(5)$ \\
C8 & $0.0296(7)$ & $0.0279(7)$ & $0.0350(8)$ & $0.0009(6)$ & $-0.0004(6)$ & $0.0023(6)$
\end{tabular}


supporting information

\begin{tabular}{lllllll} 
C9 & $0.0281(7)$ & $0.0251(7)$ & $0.0504(9)$ & $0.0042(6)$ & $0.0023(7)$ & $0.0019(7)$ \\
C10 & $0.0305(8)$ & $0.0280(8)$ & $0.0457(9)$ & $-0.0021(6)$ & $0.0105(7)$ & $-0.0069(7)$ \\
C11 & $0.0338(8)$ & $0.0325(8)$ & $0.0313(7)$ & $-0.0031(6)$ & $0.0057(6)$ & $-0.0008(6)$ \\
C12 & $0.0274(7)$ & $0.0233(7)$ & $0.0339(7)$ & $-0.0006(5)$ & $0.0038(6)$ & $0.0047(6)$ \\
\hline
\end{tabular}

Geometric parameters $\left(A,{ }^{\circ}\right)$

\begin{tabular}{|c|c|c|c|}
\hline $\mathrm{Br} 1-\mathrm{C} 2$ & $1.9044(14)$ & C6- $\mathrm{H} 6$ & 0.9500 \\
\hline $\mathrm{Se} 1-\mathrm{C} 1$ & $1.9171(14)$ & $\mathrm{C} 7-\mathrm{C} 8$ & $1.395(2)$ \\
\hline $\mathrm{Se} 1-\mathrm{C} 7$ & $1.9198(14)$ & $\mathrm{C} 7-\mathrm{C} 12$ & $1.386(2)$ \\
\hline $\mathrm{C} 1-\mathrm{C} 2$ & $1.391(2)$ & $\mathrm{C} 8-\mathrm{H} 8$ & 0.9500 \\
\hline $\mathrm{C} 1-\mathrm{C} 6$ & $1.4001(19)$ & $\mathrm{C} 8-\mathrm{C} 9$ & $1.387(2)$ \\
\hline $\mathrm{C} 2-\mathrm{C} 3$ & $1.386(2)$ & C9-H9 & 0.9500 \\
\hline $\mathrm{C} 3-\mathrm{H} 3$ & 0.9500 & $\mathrm{C} 9-\mathrm{C} 10$ & $1.386(2)$ \\
\hline $\mathrm{C} 3-\mathrm{C} 4$ & $1.387(2)$ & $\mathrm{C} 10-\mathrm{H} 10$ & 0.9500 \\
\hline $\mathrm{C} 4-\mathrm{H} 4$ & 0.9500 & $\mathrm{C} 10-\mathrm{C} 11$ & $1.384(2)$ \\
\hline $\mathrm{C} 4-\mathrm{C} 5$ & $1.383(2)$ & C11-H11 & 0.9500 \\
\hline $\mathrm{C} 5-\mathrm{H} 5$ & 0.9500 & $\mathrm{C} 11-\mathrm{C} 12$ & $1.390(2)$ \\
\hline $\mathrm{C} 5-\mathrm{C} 6$ & $1.389(2)$ & $\mathrm{C} 12-\mathrm{H} 12$ & 0.9500 \\
\hline $\mathrm{C} 1-\mathrm{Se} 1-\mathrm{C} 7$ & $99.19(6)$ & $\mathrm{C} 8-\mathrm{C} 7-\mathrm{Se} 1$ & $120.23(11)$ \\
\hline $\mathrm{C} 2-\mathrm{C} 1-\mathrm{Se} 1$ & $118.90(10)$ & $\mathrm{C} 12-\mathrm{C} 7-\mathrm{Se} 1$ & $118.99(11)$ \\
\hline $\mathrm{C} 2-\mathrm{C} 1-\mathrm{C} 6$ & $117.80(13)$ & $\mathrm{C} 12-\mathrm{C} 7-\mathrm{C} 8$ & $120.67(14)$ \\
\hline $\mathrm{C} 6-\mathrm{C} 1-\mathrm{Se} 1$ & $123.27(11)$ & $\mathrm{C} 7-\mathrm{C} 8-\mathrm{H} 8$ & 120.4 \\
\hline $\mathrm{C} 1-\mathrm{C} 2-\mathrm{Br} 1$ & $120.07(11)$ & $\mathrm{C} 9-\mathrm{C} 8-\mathrm{C} 7$ & $119.25(14)$ \\
\hline $\mathrm{C} 3-\mathrm{C} 2-\mathrm{Br} 1$ & $117.89(11)$ & $\mathrm{C} 9-\mathrm{C} 8-\mathrm{H} 8$ & 120.4 \\
\hline $\mathrm{C} 3-\mathrm{C} 2-\mathrm{C} 1$ & $122.04(14)$ & $\mathrm{C} 8-\mathrm{C} 9-\mathrm{H} 9$ & 119.9 \\
\hline $\mathrm{C} 2-\mathrm{C} 3-\mathrm{H} 3$ & 120.4 & $\mathrm{C} 10-\mathrm{C} 9-\mathrm{C} 8$ & $120.25(15)$ \\
\hline $\mathrm{C} 2-\mathrm{C} 3-\mathrm{C} 4$ & $119.24(15)$ & $\mathrm{C} 10-\mathrm{C} 9-\mathrm{H} 9$ & 119.9 \\
\hline $\mathrm{C} 4-\mathrm{C} 3-\mathrm{H} 3$ & 120.4 & $\mathrm{C} 9-\mathrm{C} 10-\mathrm{H} 10$ & 119.9 \\
\hline $\mathrm{C} 3-\mathrm{C} 4-\mathrm{H} 4$ & 120.1 & $\mathrm{C} 11-\mathrm{C} 10-\mathrm{C} 9$ & $120.16(15)$ \\
\hline $\mathrm{C} 5-\mathrm{C} 4-\mathrm{C} 3$ & $119.82(15)$ & $\mathrm{C} 11-\mathrm{C} 10-\mathrm{H} 10$ & 119.9 \\
\hline $\mathrm{C} 5-\mathrm{C} 4-\mathrm{H} 4$ & 120.1 & $\mathrm{C} 10-\mathrm{C} 11-\mathrm{H} 11$ & 119.9 \\
\hline $\mathrm{C} 4-\mathrm{C} 5-\mathrm{H} 5$ & 119.7 & $\mathrm{C} 10-\mathrm{C} 11-\mathrm{C} 12$ & $120.22(15)$ \\
\hline $\mathrm{C} 4-\mathrm{C} 5-\mathrm{C} 6$ & $120.64(15)$ & $\mathrm{C} 12-\mathrm{C} 11-\mathrm{H} 11$ & 119.9 \\
\hline $\mathrm{C} 6-\mathrm{C} 5-\mathrm{H} 5$ & 119.7 & $\mathrm{C} 7-\mathrm{C} 12-\mathrm{C} 11$ & $119.45(14)$ \\
\hline $\mathrm{C} 1-\mathrm{C} 6-\mathrm{H} 6$ & 119.8 & $\mathrm{C} 7-\mathrm{C} 12-\mathrm{H} 12$ & 120.3 \\
\hline $\mathrm{C} 5-\mathrm{C} 6-\mathrm{C} 1$ & $120.39(14)$ & $\mathrm{C} 11-\mathrm{C} 12-\mathrm{H} 12$ & 120.3 \\
\hline $\mathrm{C} 5-\mathrm{C} 6-\mathrm{H} 6$ & 119.8 & & \\
\hline $\mathrm{Br} 1-\mathrm{C} 2-\mathrm{C} 3-\mathrm{C} 4$ & $179.31(12)$ & $\mathrm{C} 4-\mathrm{C} 5-\mathrm{C} 6-\mathrm{C} 1$ & $0.5(2)$ \\
\hline $\mathrm{Se} 1-\mathrm{C} 1-\mathrm{C} 2-\mathrm{Br} 1$ & $4.17(16)$ & $\mathrm{C} 6-\mathrm{C} 1-\mathrm{C} 2-\mathrm{Br} 1$ & $-177.44(10)$ \\
\hline $\mathrm{Se} 1-\mathrm{C} 1-\mathrm{C} 2-\mathrm{C} 3$ & $-175.83(12)$ & $\mathrm{C} 6-\mathrm{C} 1-\mathrm{C} 2-\mathrm{C} 3$ & $2.6(2)$ \\
\hline $\mathrm{Se} 1-\mathrm{C} 1-\mathrm{C} 6-\mathrm{C} 5$ & $175.87(11)$ & $\mathrm{C} 7-\mathrm{C} 8-\mathrm{C} 9-\mathrm{C} 10$ & $0.9(2)$ \\
\hline $\mathrm{Se} 1-\mathrm{C} 7-\mathrm{C} 8-\mathrm{C} 9$ & $-177.21(12)$ & $\mathrm{C} 8-\mathrm{C} 7-\mathrm{C} 12-\mathrm{C} 11$ & $0.2(2)$ \\
\hline $\mathrm{Se} 1-\mathrm{C} 7-\mathrm{C} 12-\mathrm{C} 11$ & $176.40(11)$ & $\mathrm{C} 8-\mathrm{C} 9-\mathrm{C} 10-\mathrm{C} 11$ & $0.1(2)$ \\
\hline $\mathrm{C} 1-\mathrm{C} 2-\mathrm{C} 3-\mathrm{C} 4$ & $-0.7(2)$ & $\mathrm{C} 9-\mathrm{C} 10-\mathrm{C} 11-\mathrm{C} 12$ & $-1.0(2)$ \\
\hline $\mathrm{C} 2-\mathrm{C} 1-\mathrm{C} 6-\mathrm{C} 5$ & $-2.4(2)$ & $\mathrm{C} 10-\mathrm{C} 11-\mathrm{C} 12-\mathrm{C} 7$ & $0.8(2)$ \\
\hline
\end{tabular}


supporting information

$\begin{array}{llll}\mathrm{C} 2-\mathrm{C} 3-\mathrm{C} 4-\mathrm{C} 5 & -1.3(2) & \mathrm{C} 12-\mathrm{C} 7-\mathrm{C} 8-\mathrm{C} 9 & -1.1(2) \\ \mathrm{C} 3-\mathrm{C} 4-\mathrm{C} 5-\mathrm{C} 6 & 1.4(3) & & \end{array}$

